\title{
Mouse Mammary Tumor Virus in Human Breast Cancer
}

Red Herring or Smoking Gun?

\author{
Andrew L. Mason, ${ }^{\star}$ Susanna Y. Gilady, ${ }^{*}$ and \\ John R. Mackey ${ }^{\dagger}$ \\ From the Division of Gastroenterology,* Department of Medicine, \\ and the Department of Medical Oncology, ${ }^{\dagger}$ Cross Cancer \\ Institute, University of Alberta, Edmonton, Alberta, Canada
}

Breast cancer is a complex disease that arises from the interactions of genetic predisposition with environmental, hormonal, and lifestyle factors. Despite growing appreciation of the molecular pathways underlying breast carcinogenesis and the heterogeneity of the disease, most patients with sporadic breast cancer lack clear etiologic risk factors. Although the process of breast carcinogenesis is histologically recognized as a spectrum of disorders including normal human breast epithelium, hyperplasia, atypia, carcinoma in situ, and invasive and metastatic carcinomas, the contribution of virus infection to initiation and promotion of mammary carcinogenesis remains uncertain.

\section{Breast Cancer Initiation: A Role for Mouse Mammary Tumor Virus?}

Mouse mammary tumor virus (MMTV) was first linked to human breast cancer 50 years ago. ${ }^{1}$ Today, however, the hypothesis that MMTV is etiologically linked to human breast cancer holds little traction with virologists or clinicians, for reasons that will be summarized later. Nevertheless, a few groups have persevered with study of the MMTV hypothesis as a human pathogen associated with breast cancer. In this issue of The American Journal of Pathology, Mazzanti and colleagues ${ }^{2}$ sought evidence of MMTV infection in carefully selected human breast tissues ranging from normal benign epithelial elements through preinvasive and invasive carcinomas. With use of laser microdissection and appropriate techniques to address the risk of contamination and false-positive signals, that team reported detection of nucleic acid sequences resembling MMTV in human preinvasive lesions by means of both fluorescent nested PCR and chromogenic in situ hybridization. Tumor samples from ductal carcinoma in situ demonstrated the highest viral load and frequency of sequences resembling MMTV (80\%), whereas $27 \%$ of atypical ductal hyperplasia samples exhibited evidence of infection, compared with 35\% of those with infiltrating ductal carcinoma. ${ }^{2}$ The implication of these findings is that a virus similar to MMTV may be involved in initiation of human breast cancer.

\section{MMTV and Cancer: The Backstory}

To contextualize these findings, it is necessary to address the history of MMTV and its potential link to human breast cancer. In 1936, Joseph Bittner identified a filterable "extrachromosomal" factor that led to development of breast cancer in mice. ${ }^{3}$ Subsequently, MMTV was characterized at electron microscopy as a type B viral particle. The agent was then demonstrated to have reverse transcriptase activity, similar to other retroviruses, and hormone-responsive elements in the viral genome that enhance viral replication during pregnancy. MMTV was once extensively studied as a prototypic tumor virus with an important role in revealing fundamental aspects of viral pathogenesis. The elemental observation that insertion of the MMTV proviral genome resulted in activation of proto-oncogenes was celebrated with the award of the Nobel Prize to Harold Varmus in 1989. ${ }^{3}$ In addition, viral superantigens were first characterized in MMTV. This mechanism is required for stimulating lymphocyte proliferation, which is essential for viral replication and spread. ${ }^{3}$ Of note, MMTV was one of the first mammalian viruses observed to induce cancer.

Supported by the Alberta Heritage Foundation for Medical Research, the Canadian Institutes for Health Research, and the Canadian Liver Foundation

Accepted for publication August 12, 2011.

CME Disclosure: None of the authors disclosed any relevant financial relationships.

Address reprint requests to Andrew L. Mason, MBBS, MRCPI, Division of Gastroenterology, University of Alberta, 7-142J, KGR, Edmonton, AB, Canada. E-mail: andrew.mason@ualberta.ca. 


\section{Support Lost and Regained}

In 1971, Moore and colleagues ${ }^{1}$ reported evidence of type B particles resembling MMTV in the milk of $60 \%$ of patients with breast cancer. Soon thereafter, numerous reports emerged that demonstrated humoral and cellular reactivity to MMTV, nucleic acid hybridization studies with MMTV sequences, and reverse transcriptase activity in breast cancer samples. However, an equal and opposite reaction soon emerged from virologists unable to find evidence of MMTV infection in patients with breast cancer. Questions were asked about the obvious differences in pathologic features of breast cancer in humans compared with those in mice and whether the positive findings could be attributable to artifact and the presence of endogenous retroviruses. The debate waged on, and eventually camps polarized into those who did or did not believe in the existence of MMTV infection in humans. ${ }^{3}$ Indeed, the discussion became personalized, and MMTV was soon laughably dubbed a "rumor virus" because, at the time, retroviruses were referred to as "tumor viruses." ${ }^{4}$ The appeal of studying MMTV in humans diminished by the 1980s after HIV emerged as a major human pathogen and the funds to study MMTV disappeared.

Interest in a viral etiology of breast cancer was rekindled, however, in the 1990s, when multiple groups used PCR and reported the presence of sequences resembling MMTV in patients with breast cancer. ${ }^{5-8}$ The reported prevalence of MMTV infection in breast cancer samples varied geographically, with a variable median range of approximately $30 \%$ to $50 \% .^{9}$ Then history was repeated, with many competing researchers reporting their inability to detect MMTV sequences using PCR on human breast cancer samples. ${ }^{9,10}$

\section{Argument for MMTV in Breast Cancer}

What argues against the MMTV hypothesis of breast cancer? In addition to the inability of some laboratories to detect MMTV sequences in breast cancer, doubts were expressed about the biology of MMTV as a human pathogen. Some of these biological contradictions seem to have been addressed. Despite the contention that human tissues lack receptors for MMTV, ${ }^{10}$ Indik and colleagues ${ }^{11,12}$ have demonstrated infection and spread of MMTV in human cells in vitro. Some have argued that the viral etiology of breast cancer would predict an increased incidence of human breast cancers in states of immunosuppression, ${ }^{10}$ whereas the opposite seems to occur because organ transplant recipients demonstrate a lower incidence of breast cancer. ${ }^{13}$ This observation, however, remains compatible with the natural biology of MMTV because there is an absolute requirement for lymphocytes to traffic the virus. As a result, immunosuppression limits viral spread and reduces the incidence of breast cancer in mice with MMTV infection. ${ }^{13}$ The recognition that the human genome contains endogenous retroviral sequences and the possibility of cross-contamination when performing nested PCR remain frequent criticisms of the published literature. Nonetheless, there is sufficient diversity of the MMTV genome and its closest relative in humans, ie, HERV-K (human endogenous retrovirus $\mathrm{K}$ ), to differentiate them using PCR. It is also possible that MMTV infection is simply an epiphenomenon without causal relevance in which breast epithelial cells undergoing malignant transformation are more likely to become infected.

Several additional lines of circumstantial evidence support an association between MMTV and human breast cancer. What is the epidemiologic evidence? First, mouse exposure is common. One study reported that $95 \%$ of innercity homes demonstrated evidence of mouse deposits; a finding that was irrespective of income. ${ }^{14}$ Stewart and colleagues ${ }^{15}$ reported that the incidence of breast cancer correlated with the geographic distribution of various species of wild mice. The common house mouse, Mus domesticus, as compared with Mus musculus, is thought to shed a higher viral burden of MMTV. ${ }^{15}$ The former is the predominant species in Western Europe and North America, where the highest prevalences of breast cancer are found, whereas the latter species resides in countries with low prevalence of breast cancer. ${ }^{15}$ This distribution also roughly parallels the frequency of MMTV sequences reported in breast cancer. MMTV is seldom, if ever, detected in breast cancer samples from Japan, China, and Vietnam, as compared with approximately $40 \%$ in the Western world, notwithstanding the negative results from several centers in Europe and North America. ${ }^{9,10}$

One could argue by analogy that MMTV is related to breast carcinogenesis. Viral infection is accepted as a causative agent in several other human cancers including uterine and cervical cancer, lymphoma, hepatoma, and oropharangeal carcinoma. It is also recognized that many viruses including the herpesviridae and retroviridae are passed through human breast milk, and in these examples, viral infection must become established in mammary glands to be secreted into milk. There are also instances of other viral agents linked to breast cancer in other species such as primates and mice. Furthermore, other viruses linked to breast cancer in humans include known oncogenic agents such as Epstein-Barr virus and papillomaviruses. ${ }^{9}$

In addition, agents resembling MMTV have been linked to other diseases in humans. In 2003, a human betaretrovirus with $95 \%$ homology to MMTV was characterized in patients with primary biliary cirrhosis, an autoimmune liver disease. ${ }^{16}$ While this association is controversial, the findings stirred some interest because the virus triggered a disease-specific phenotype in vitro that was associated with development of autoimmunity, and MMTV infection was associated with the same phenotype in mice and in humans. ${ }^{16,17}$ More recently, proof of viral infection was demonstrated by detecting integration of the MMTV nucleotide sequences in human DNA using linker-mediated PCR coupled with second-generation sequencing to increase sensitivity. MMTV has also been linked to autoimmune biliary disease in several mouse models that have subsequently been used to discover novel antiretroviral therapies to treat primary biliary cirrhosis. Significant biochemical and histologic responses have been observed in humans in pilot studies that used zidovudine and lamivudine, and case reports have demonstrated complete normalization of liver test results after highly active antiviral therapy. ${ }^{17}$ Inasmuch as various studies have reported conflicting results, it is uncertain whether patients with primary biliary cirrhosis 
have a higher or lower prevalence of breast cancer. However, the biological features of MMTV fit with other aspects of primary biliary cirrhosis because the disease occurs predominantly in female patients and female hormones stimulate MMTV replication, in particular during pregnancy.

\section{Argument Against MMTV in Breast Cancer}

There remain two major criticisms that should be addressed concerning MMTV and human breast cancer. First, the virus is found only at the level of detection of PCR, and the field has yet to move on from conducting PCR studies with the intrinsic uncertainty of the results. Of note, low-level infection is consistent with the biologic features of many retroviruses including betaretroviruses such as MMTV, gammaretroviruses, and deltaretroviruses such as human T-lymphotropic virus-1 (HTLV-1). Unlike HIV, these viruses are rarely detected in serum, and they traffic in lymphocytes. For example, HTLV- 1 is detected in as few as $0.1 \%$ of peripheral blood mononuclear cells in patients with morbid disease, which is comparable to the amount of MMTV in human blood. However, HTLV-1 is accepted as a pathogen, whereas MMTV is not. The major link of HTLV-1 to development of hematologic cancer and neurologic disease was made by correlating the clustering of HTLV-1 in Japan and then in the Caribbean. ${ }^{18}$ HTLV-1 causes adult T-cell leukemia in less than $5 \%$ of infected patients, and myelitis in less than $1 \%$. These data strongly suggest that other factors may be required for development of HTLV-1-related disease, such as infection in childhood and genetic predisposition.

\section{Koch's Postulates and Hill's Criteria Considered}

So how can we determine whether MMTV in breast cancer is a red herring or a smoking gun? Usually, multiple layers of proof are required to demonstrate a definitive etiologic association of an infectious agent and disease. Koch's postulates were originally formulated for acute disease and were not directly applicable to the years-long process of carcinogenesis. However, it is interesting that some of Koch's postulates have been demonstrated retrospectively in isolated patients with HTLV-1; the virus has been detected in humans, and disease has been transmitted via blood transfusion. More relevant to this discussion are Hill's criteria of causation. ${ }^{19}$ These encompass multiple factors including the strength, consistency, and specificity of association, and the temporality and biological gradient of exposure (greater exposure leads to more disease), the plausibility and coherence of the hypothesis, and experimental data. ${ }^{19}$

If we are to follow the lead of Hill's criteria and modified Koch's postulates with MMTV in breast cancer, we have a long way to go. A critically important step would be demonstration of MMTV integration sites into DNA of human breast cancer cells; similar preliminary data are now available for primary biliary cirrhosis. Finding integration sites in genetic loci triggering oncogenic pathways would strengthen the argument for a causative role. Isolation of infectious viral particles from breast epithelial cells would be persuasive. Such data would provide the impetus to develop sensitive and specific serologic diagnostic tests to evaluate the epidemiologic relationships between MMTV and breast cancer. Were seropositivity to track with breast cancer biology, with either a molecular subtype or clinicopathologic features, the argument for causation would be further strengthened. In due course, one could envision, as the ultimate demonstration of a causative link, a vaccination program in unexposed individuals or prepubescent girls that reduced breast cancer incidence. The findings of Mazzanti and colleagues suggest that further investigation is much warranted.

\section{References}

1. Moore DH, Charney J, Kramarsky B, Lasfargues EY, Sarkar NH Brennan MJ, Burrows JH, Sirsat SM, Paymaster JC, Vaidya AB: Search for a human breast cancer virus. Nature 1971, 229:611-615

2. Mazzanti CM, Al-Hamad M, Fanelli G, Scatena C, Zammarchi F Zavaglia K, Lessi F, Pistello M, Naccarato AG, Bevilacqua G: A murine mammary tumor virus (MMTV) env-like exogenous sequence is strictly related to progression of human sporadic breast carcinoma. Am J Pathol 2011, 179:2083-2090

3. Mason A: Is there a breast cancer virus? Ochsner J 2000, 2:36-39

4. Voisset C, Weiss RA, Griffiths DJ: Human RNA "rumor" viruses: the search for novel human retroviruses in chronic disease. Microbiol Mol Biol Rev 2008, 72:157-196

5. Etkind PR, Stewart AF, Dorai T, Purcell DJ, Wiernik PH: Clonal isolation of different strains of mouse mammary tumor virus-like DNA sequences from both the breast tumors and non-Hodgkin's lymphomas of individual patients diagnosed with both malignancies. Clin Cancer Res 2004, 10:5656-5664

6. Szabo S, Haislip AM, Traina-Dorge V, Costin JM, Crawford BE II, Wilson RB, Garry RF: Human, rhesus macaque, and feline sequences highly similar to mouse mammary tumor virus sequences. Microsc Res Tech 2005, 68:209-221

7. Wang Y, Holland JF, Bleiweiss IJ, Melana S, Liu X, Pelisson I, Cantarella A, Stellrecht K, Mani S, Pogo BG: Detection of mammary tumor virus env gene-like sequences in human breast cancer. Cancer Res 1995, 55:5173-5179

8. Ford CE, Tran D, Deng Y, Ta VT, Rawlinson WD, Lawson JS: Mouse mammary tumor virus-like gene sequences in breast tumors of Australian and Vietnamese women. Clin Cancer Res 2003, 9:1118-1120

9. Amarante MK, Watanabe MA: The possible involvement of virus in breast cancer. J Cancer Res Clin Oncol 2009, 135:329-337

10. Mant $\mathrm{C}$, Cason J: A human murine mammary tumour virus-like agent is an unconvincing aetiological agent for human breast cancer. Rev Med Virol 2004, 14:169-177

11. Indik S, Günzburg WH, Kulich P, Salmons B, Rouault F: Rapid spread of mouse mammary tumor virus in cultured human breast cells. Retrovirology 2007, 4:73

12. Indik S, Günzburg WH, Salmons B, Rouault F: Mouse mammary tumor virus infects human cells. Cancer Res 2005, 65:6651-6659

13. Stewart T, Tsai SC, Grayson H, Henderson R, Opelz G: Incidence of de-novo breast cancer in women chronically immunosuppressed after organ transplantation. Lancet 1995, 346:796-798

14. Phipatanakul W, Eggleston PA, Wright EC, Wood RA; the National Cooperative Inner-City Asthma Study: Mouse allergen. I: the prevalence of mouse allergen in inner-city homes. J Allergy Clin Immunol 2000; 106:1070-1074

15. Stewart TH, Sage RD, Stewart AF, Cameron DW: Breast cancer incidence highest in the range of one species of house mouse, Mus domesticus. Br J Cancer 2000, 82:446-451

16. Xu L, Shen Z, Guo L, Fodera B, Keogh A, Joplin R, O'Donnell B, Aitken J, Carman W, Neuberger J, Mason A: Does a betaretrovirus infection trigger primary biliary cirrhosis?. Proc Natl Acad Sci USA 2003, 100:8454-8459

17. Mason AL: The evidence supports a viral aetiology for primary biliary cirrhosis. J Hepatol 2011, 54:1312-1314

18. Roman GC, Navarro-Roman LI: The discovery of HTLV-1 myelitis: 21 years later. Lancet Neurol 2007, 6:104-105

19. Hill AB: The environment and disease: association or causation? Proc R Soc Med 1965, 58:295-300 